Original Article

\title{
PREPARATION AND IN VITRO EVALUATION OF MONTELUKAST SODIUM ORAL NANOEMULSION
}

\author{
YASSER Q. ALMAJIDI ${ }^{*}$, ZAINAB H. MAHDI ${ }^{2}$, NIDHAL K. MARAIE ${ }^{2}$
}

${ }^{1}$ Department of Pharmaceutics, Baghdad College of Medical Sciences, Baghdad, Iraq, 'Department of Pharmaceutics, College of Pharmacy, Mustansiriyah University, Baghdad, Iraq

Email: yasser.kasim@yahoo.com

Received: 08 Jul 2018, Revised and Accepted: 01 Aug 2018

\begin{abstract}
Objective: Oral nanoemulsion (NE) represent one of the newest technology to enhance intestinal drug permeability, bioavailability and facilitate swallowing of the oral dosage form.

Methods: In this study, montelukast sodium (MS) nanoemulsions (NEs) were formulated by ultra-sonication using different surfactants (tween 20, tween 60 and tween 80) in different surfactant: co-surfactant (ethanol) ratios (Smix). The prepared NEs were evaluated for different parameters including droplet size (DS) using zetasizer as a function of ultra-sonication time, dispersibility, phase separation, conductivity, percent transmittance, optical transparency, in vitro release in addition to morphology using transmission electron microscopic (TEM).

Results: The results revealed that F3 was the optimum formula having an average DS $32.95 \pm 2.8 \mathrm{~nm}$ after 5 min ultra-sonication assured by zetasizer and TEM, furthermore, a clear to bluish NE was formed after aqueous dilution with high conductivity $(59.2 \pm 1.76 \mu \mathrm{s} / \mathrm{cm}) \mathrm{which}$ indicated the formation of $\mathrm{O} / \mathrm{W}$ NE. In addition, an optically clear NE was formed with $(88.6 \pm 2.1) \%$ transmittance with no sedimentation, creaming or separation after centrifugation signifying the formation of a stable NE. Finally, F3 showed faster dissolution rate $(92.45 \% \pm 1.66)$ after 30 min compared to other formulas.
\end{abstract}

Conclusion: The net result of this study is the formulation of a stable oral NE containing MS which presents new easily swallowed dosage form that may enhance drug permeability as well as it may reduce drug metabolism leading to improving bioavailability for asthmatic patients.

Keywords: Montelukast sodium, Nanoemulsion, Oleic acid, Ultrasonication

(C) 2018 The Authors. Published by Innovare Academic Sciences Pvt Ltd. This is an open access article under the CC BY license (http://creativecommons.org/licenses/by/4.0/) DOI: http://dx.doi.org/10.22159/ijap.2018v10i5.28367

\section{INTRODUCTION}

Although oral administration is the most preferred route for administration of pharmaceuticals, it is restricted by swallowing difficulty of the solid dosage form in addition to dissolution, absorption and bioavailability problems $[1,2]$.

For many years, numerous technologies were developed to produce easily swallowed dosage forms and to enhance its oral bioavailability, from which oral nanoemulsion (NE) was one of the most promising investments $[3,4]$.

Nanoemulsions (NEs) are thermodynamically stable dispersion of oil and water with deformable droplets in the range of (10-200 nanometre "nm") which have specific physicochemical properties such as low viscosity, transparency, optical isotropy and does not have the tendency to coalesce due to the presence of a stabilizing surfactant: co-surfactant mixture. Their small elastic droplets give them higher stability because the diffusion rate is higher than the sedimentation or creaming rate induced by the gravity force. $\mathrm{NE}$ are classified according to the composition of the dispersed phase and dispersion media into three types oil in water $(\mathrm{O} / \mathrm{W})$, water in oil $(\mathrm{W} / \mathrm{O})$ and bicontinuous, were each possess certain applications [5-8].

Montelukast is a selective leukotriene receptor antagonist used for the maintenance treatment of asthma, chronic asthma attacks and to relieve symptoms of seasonal allergies. Although montelukast is marketed as the sodium salt in the form of tablets and inhalers, no liquid dosage form is available because of the stability problem of this salt in the aqueous media $[9,10]$.

For this reason, this work was adapted to formulate a new oral dosage form of montelukast sodium (MS) as nanoemulsion in order to produce easily swallowed dosage form that may improve its absorption and bioavailability.

\section{MATERIALS AND METHODS}

\section{Materials}

Tween 20, tween 40, tween 60, tween 80, propylene glycol (PG), polyethylene glycol (PEG) 200 and 400 (J. T Baker, China), oleic acid, clove oil, lemon oil, cardamom oil, olive oil and orange flavor $(\mathrm{CDH}$, India), montelukast sodium (MS) (Sigma Chemical Co. Aldrich, USA), deionized distilled water (DDW) was used for all experiments. All other reagents, chemicals and solutions used were of analytical grade.

\section{Construction of phase diagrams}

Pseudo-ternary phase diagrams were constructed by aqueous titration method to examine the formation of $\mathrm{O} / \mathrm{W} \mathrm{NE}$ using four components: oil (oleic acid), surfactant (tween 80, tween 60, and tween 20), co-surfactant (ethanol), and an aqueous phase. The diagrams of triangular coordinate were constructed using different combination of surfactant/co-surfactant mixtures (Smix) including (tween 80: ethanol, tween 60: ethanol and tween 20: ethanol) at the desired ratios $(1: 1,1: 2,2: 1)$ as listed in the table (1). Emulsion area boundaries were programed using different ratios of oil to Smix ranging from (1:9 to 9:1). As DDW was added drop by drop to the mixture of oleic acid with Smix under gentle stirring. If turbidity appeared, followed by phase separation, the samples were considered to be biphasic. If clear and transparent mixtures were visualized after stirring, the samples were considered monophasic. The samples were marked as points in the phase diagram. The area covered by these points was considered to be the nanoemulsion region of existence $[11,12]$. The larger the area of the emulsion in the phase diagram was selected as the best NE composition for further study because of its better hydration capacity.

\section{Preparation of montelukast nanoemulsion}

Nanoemulsions were prepared by the ultra-sonication method. The composition of the NE was chosen according to the pseudo-ternary phase diagram. MS powder (4 mg) was dissolved in the selected oil, surfactant and co-surfactant mixture was added in the chosen concentration, and flavored DDW (with $1 \%$ orange flavor) was added dropwise with continuous stirring until clear NE was formed, this was followed by utra-sonication to obtain very small droplet nanoemulsion. The final concentration of MS in the NE was $4 \mathrm{mg} / \mathrm{ml}$ as shown in table $1[13,14]$. 
Table 1: Composition of montelukast sodium NE

\begin{tabular}{|c|c|c|c|c|c|c|c|}
\hline Formula & Surfactant & Co-surfactant & S./Cos. ratio & Oleic acid oil \%v/v & Smix. \%v/v & Flavored DDW*\%v/v & Drug mg/ml \\
\hline F1 & Tween80 & Ethanol & $1: 1$ & 10 & 60 & 30 & 4 \\
\hline $\mathrm{F} 2$ & Tween 60 & Ethanol & $1: 1$ & 10 & 60 & 30 & 4 \\
\hline F3 & Tween 20 & Ethanol & $1: 1$ & 10 & 60 & 30 & 4 \\
\hline F4 & Tween20 & Ethanol & $1: 2$ & 10 & 60 & 30 & 4 \\
\hline F5 & Tween 20 & Ethanol & $2: 1$ & 10 & 60 & 30 & 4 \\
\hline F6 & Tween 20 & Ethanol & $1: 1$ & 15 & 60 & 25 & 4 \\
\hline
\end{tabular}

*All NE formulas contain $1 \%$ orange flavour, ${ }^{*}$ Abbreviations: S./Cos. ratio, surfactant/co-surfactant ratio; Smix \%, percentage of the surfactant and co-surfactant in the NE; DDW, deionized distilled water.

\section{Characterization of montelukast sodium NE}

\section{Saturated solubility study}

The saturated solubility of MS was performed in different oils including oleic acid, clove oil, lemon oil, cardamom oil, olive oil in addition to the orange flavor. Furthermore, the study also performed on different surfactants including tween 20, tween 40, tween 60 , tween 80, PG, PEG 200 and 400 in addition to different co-surfactants including ethanol, isopropanol, and butanol. This was performed by shaking an excess amount of MS to $2 \mathrm{ml}$ of each of the previous liquid at $25 \pm 10{ }^{\circ} \mathrm{C}$ for $24 \mathrm{~h}$, finally the supernatant layer was removed filtered and analyzed using UV-spectrophotometer at $285 \mathrm{~nm}[15,16]$.

\section{Droplet size versus ultra-sonication time}

The ultra-sonication time was optimized according to droplet size measurement for every formula after different ultra-sonication intervals (1, 2, 3, 5 and $10 \mathrm{~min}$ ). The evaluation of droplet size was performed using Nano Zetasizer for each sample, different dilutions were performed and the test was done in triplicate to find the mean droplet size at $20^{\circ} \mathrm{C}[17]$.

\section{Dispersability test}

Dilution or dispersibility test was performed to assess the physical stability of the prepared NEs, since $\mathrm{O} / \mathrm{W}$ NE is dilutable with water, whereas $\mathrm{W} / \mathrm{O}$ are not and undergo phase inversion into $0 / \mathrm{W}$ NE. This test can be done by taking $1 \mathrm{ml}$ of the prepared NE (F1-F6) and diluted it in $500 \mathrm{ml}$ of distilled water with constant stirring at $50 \mathrm{rpm}$ at $37^{\circ} \mathrm{C}$ and observed for clarity, turbidity or phase separation $[18,19]$.

\section{Phase separation}

NE formulas (F1-F6) were assessed for physical stability by centrifugation at $10,000 \mathrm{rpm}$ for a period of $30 \mathrm{~min}$ and examined for any phase separation [20].

\section{Conductance test}

O/w NEs, where the external phase is water, are highly conducting, whereas $\mathrm{W} / 0$ are not, since water is the internal or dispersal phase. To determine the nature or type of the continuous phase and to detect phase inversion phenomena the electrical conductivity $(\sigma)$ has to be measured and this was done by using a conductometer via laying the conductometer probe erect in $10 \mathrm{ml}$ of the prepared formula in a beaker at room temperature, and the apparatus will register the results in $\mu \mathrm{s} / \mathrm{cm}[21]$.

\section{Percentage transmittance}

Percentage transmittances (\%T) of NE prove the transparency of the systems. For measurement of percentage transmittance $(\% \mathrm{~T})$, NE formulas were diluted 10 times with distilled water and \% $\mathrm{T}$ was checked against distilled water using a visible spectrophotometer. The percent transmittance of the all formulations was measured at $285 \mathrm{~nm}$ using this equation [22]:

$A=2-\log \% T$

Where;

A: absorbance

$\% \mathrm{~T}$ : transmittance percentage

\section{Optical transparency}

Optical transparency of the formulas was determined by visually inspecting the sample in a clear and transparent container under the presence of good light against reflection into the eyes, and viewed against black and white illuminated background [23].

\section{In vitro drug release studies}

The release of MS from NEs carried out by using dialysis bag MWCO (2000 Da). $10 \mathrm{ml}$ MS nanoemulsion containing $40 \mathrm{mg}$ of the drug was placed into dialysis bag. USP II rotating paddle apparatus was used to measure the in vitro drug release of all formulas. The dissolution medium $(900 \mathrm{ml}$ of $0.1 \mathrm{~N} \mathrm{HCl})$ was placed into the release jar maintaining the speed of $100 \mathrm{rpm}$ and temperature at $37 \pm 0.5^{\circ} \mathrm{C}$. Release studies were carried out for $2 \mathrm{~h}$. Five ml of aliquot is withdrawn at an interval of 5, 10, 20, 30, 45, 60, 90,120 min. After collecting the sample, the dissolution medium was replenished with the same volume of fresh medium, and the sample was filtered. The samples were analyzed at 285 nm by UV-visible spectrophotometer [24].

\section{Transmission electron microscopic analysis}

Morphology of droplets was observed using transmission electron microscopic (TEM). One drop of diluted NE (100 times) was placed on a 200-mesh film grid and dried at room temperature; then samples were stained using uranyl acetate and allowed to dry for 10 min before observation with the electron microscope [25].

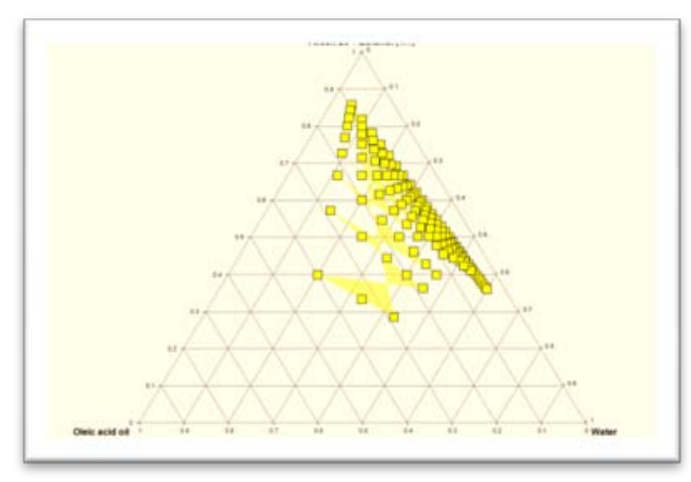

(A)

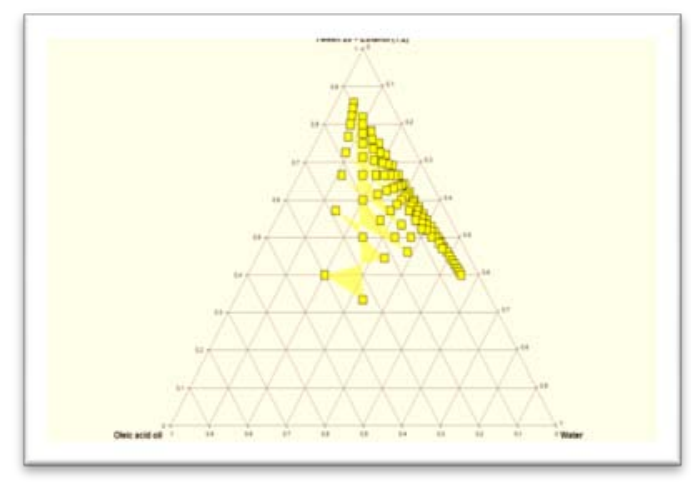

(B) 


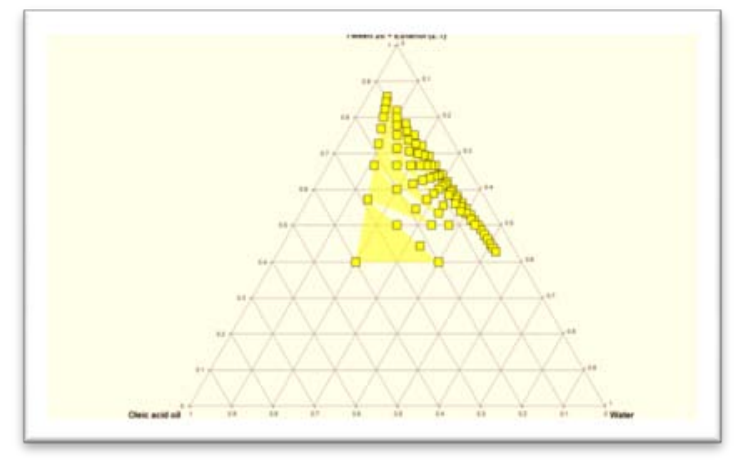

(C)

Fig. 1: Pseudo-ternary phase diagram of montelukast sodium $0 / W$ NE formulated using (A) oleic acid, tween20: ethanol

(Smix 1:1) and DDW, (B) oleic acid, tween: ethanol (Smix 1:2) and DDW, (C) oleic acid, tween20: ethanol (Smix 2:1) and DDW

\section{RESULTS AND DISCUSSION}

\section{Construction of phase diagrams}

Fig. 1 clearly signifies the pseudo-ternary phase diagram of MS NE constructed using oleic acid and DDW with tween 20 (surfactant) and ethanol (co-surfactant) at different Smix ratios. It is obvious that fig. 1(A) composed from 1:1 Smix shows the largest stable NE area. Tween 20 is a non-ionic surfactant with HLB equals 16.7, having a hydrophilic head and ahydrophobic tail, it is used to reduce the interfacial tension ( $\mathrm{\gamma}$ ) between oleic acid (oil phase) and water by formation of the protective film surrounding the oil droplet, thus reducing contact between oil globules and prevent coalescence. On the other hand, ethanol is used as a short chain co-surfactant for further reduction of $(\mathrm{y})$ and also to reduce viscosity and increase the mobility of hydrophobic tail to enhance more penetration on the interphase $[26,27]$.

\section{Evaluation of montelukast nanoemulsion}

\section{Saturated solubility results}

Fig. (2) Clearly demonstrates the solubility of MS in different oil, surfactants and co-surfactants. The significantly higher solubility of the drug in orange flavor compared to other oils could be explained to the higher aqueous solubility of the flavors comparing to the oils [28]. On the other hand, the drug possesses higher solubility in tween 20due to the higher HLB value of (16.7) and hence greater hydrophilicity compared to the other grades having lower HLB values $(15.6,15,14.9)$ for tween40, tween80, tween60 respectively [29]. Furthermore, the significantly higher solubility of the drug in the cosurfactant is arranged in the order PEG 400>PEG 200> ethanol>Propanol>isobutanol which might be due the higher molecular weight and hydrophilicity of PEG 400 compared to the others [30].

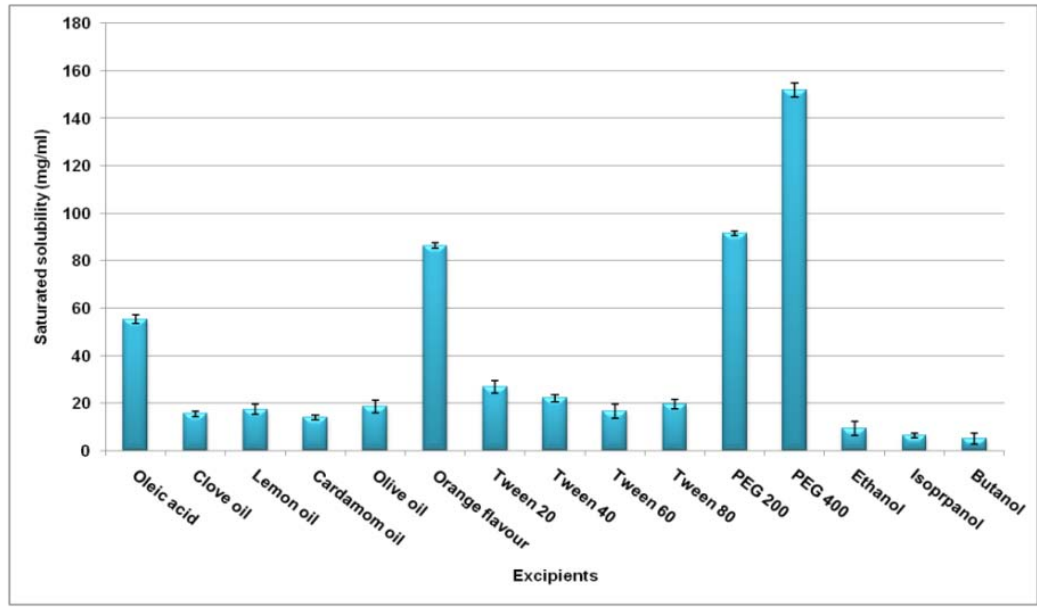

Fig. 2: Saturated solubility of montelukast sodium in different excipients (Results are expressed as mean \pm SD, $n=3$ )

\section{Droplet size and ultra-sonication time evaluation}

Regarding ultra-sonication time, mean droplet size for each formula was evaluated and the results are demonstrated in the table (2). The data showed a significant reduction in droplet size after increasing ultra-sonication time for each formula from 2 to 5 min with the disappearance of the droplets after 10 min indicating destruction of the internal phase globule after this period. For this reason, ultrasonication was fixed at $5 \mathrm{~min}$ in the preparation of montelukast NEs [31].

Table 2: Mean droplet size in $\mathrm{nm}$ as a function of ultra-sonication time

\begin{tabular}{llllll}
\hline Formula & DS/1 min & DS/2 $\min$ & DS/3 $\min$ & DS/5 $\min$ & DS/10 $\mathbf{m i n}$ \\
\hline F1 & $204.48 \pm 1.3$ & $140.56 \pm 3.87$ & $97.73 \pm 3.74$ & $64.56 \pm 1.4$ & - \\
F2 & $289.86 \pm 3.07$ & $147.32 \pm 4.25$ & $86.57 \pm 1.04$ & $78.46 \pm 1.07$ & - \\
F3 & $276.66 \pm 2.99$ & $99.83 \pm 2.98$ & $74.02 \pm 4.98$ & $32.95 \pm 2.8$ & - \\
F4 & $347.89 \pm 1.98$ & $104.04 \pm 1.04$ & $87.64 \pm 3.98$ & $48.40 \pm 1.03$ & - \\
F5 & $367 \pm 33 \pm 5.26$ & $159.02 \pm 2.76$ & $98.53 \pm 1.04$ & $77.98 \pm 3.76$ & -- \\
F6 & $398.64 \pm 2.76$ & $176.05 \pm 4.95$ & $102.34 \pm 2.8$ & $86.45 \pm 4.76$ & - \\
\hline
\end{tabular}

*Abbreviations: DS, mean droplet size; min, minutes. Results are expressed as mean \pm SD, $n=3$

\section{Dilution test}

All prepared NEs (F1-F6) gives a clear to clear bluish NE when diluted with water. This indicates that all of them are O/W Nano emulsion [32].

\section{Phase separation}

All prepared NEs were subjected to centrifugation at $3500 \mathrm{rpm}$ for a period of $30 \mathrm{~min}$ and examined for any change in phase separation. The result indicates there is no creaming, sedimentation or phase 
separation upon centrifugation for all the prepared NE formulas was observed, this indicates their stability. This proves that the thermal motion of the droplets (Brownian motion) exceeds the external forces such as gravitation or centrifugation [33, 34].

\section{Conductance test}

Measuring the electrical conductivity using a conductometer for the selected formula (F3). The result showed a high conductivity $(59.2 \pm 1.76 \mu \mathrm{s} / \mathrm{cm})$ proving that formula was $0 / \mathrm{W}$ because of the high conductivity of water. The higher conductivity of $\mathrm{NE}$ is attributed to a large percentage of water which allows more freedom for mobility of ions [35].

\section{Percentage transmittance}

The turbidity of the NEs was measured using UV absorbance at 285 $\mathrm{nm}$. 1The higher absorbance (more turbidity) means lower \% transmittance. Percentage transmittance of NEs proves the translucence of the system. The results of percent transmittance indicated that all the prepared formulations were translucent [36], as shown in table 3.

Table 3: Percentage transmittance $(\% \mathrm{~T})$ of prepared nanoemulsion formulations

\begin{tabular}{ll}
\hline Formula & Percentage transmittance (\%T) \\
\hline F1 & $94.23 \pm 1.5$ \\
F2 & $90.3 \pm 1.8$ \\
F3 & $88.6 \pm 2.1$ \\
F4 & $92.7 \pm 1.1$ \\
F5 & $90.2 \pm 1.7$ \\
F6 & $87.5 \pm 1.4$ \\
\hline
\end{tabular}

*Results are expressed as mean $\pm \mathrm{SD}, \mathrm{n}=3$

\section{Optical transparency}

All the prepared NE formulas were optically clear. Smallest droplet size causes weak light scatters (more transparent), and this indicated nano-sized emulsion [37].

\section{In vitro dissolution study}

Results of in vitro release from montelukast NE were shown in fig. (3). The fig. clearly demonstrated a significant increment on drug release from F1-F3 formulated using Tween 80, Tween 60 and Tween 20 respectively as a surfactant, with a greater degree of release represented by $\mathrm{F} 3$ from all other formulas $(92.45 \% \pm 1.66$ after $30 \mathrm{~min}$ ). This can be explained due to the higher HLB value of
Tween 20 (16.7) comparing to the other surfactants that enhanced the solubility and the dissolution of the drug from F3 [38] Furthermore, a high \% of drug release with a non-significant difference was observed in F4 and F5 formulated by using different surfactant: co-surfactant ratios 1:2 and 2:1 could be explained to the greater reduction in the surface tension leading to greater hydration and smaller droplet size and consequently greater dissolution [39].

\section{Transmission electron microscopic (TEM) analysis}

The result of TEM of the optimal formula (F3) demonstrated discrete dark globules with bright surroundings with an average diameter of $32.95 \mathrm{~nm}$ as shown in fig. 4 .

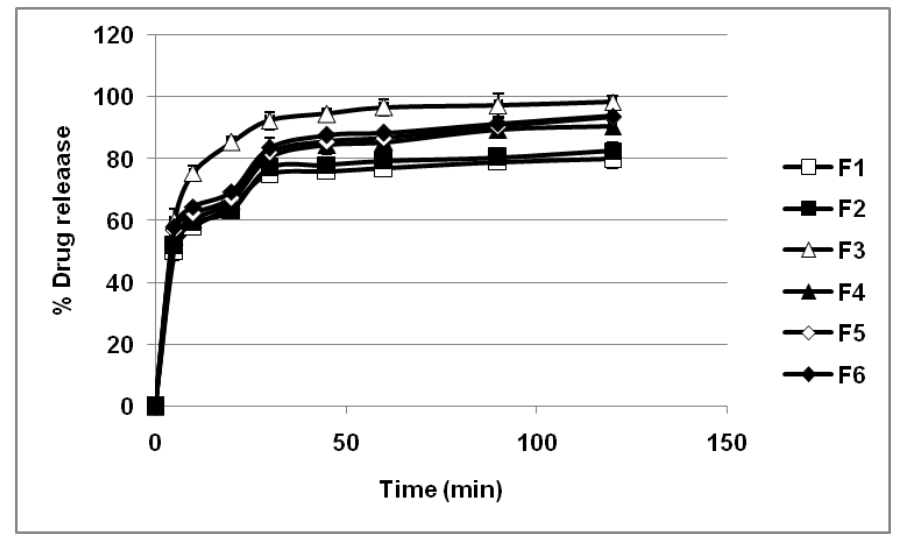

Fig. 3: In vitro release profile for montelukast $\mathrm{NE}$ (F1-F6) in $0.1 \mathrm{~N} \mathrm{HCl}$ (Results are expressed as mean $\pm \mathrm{SD}, \mathrm{n}=3$ )
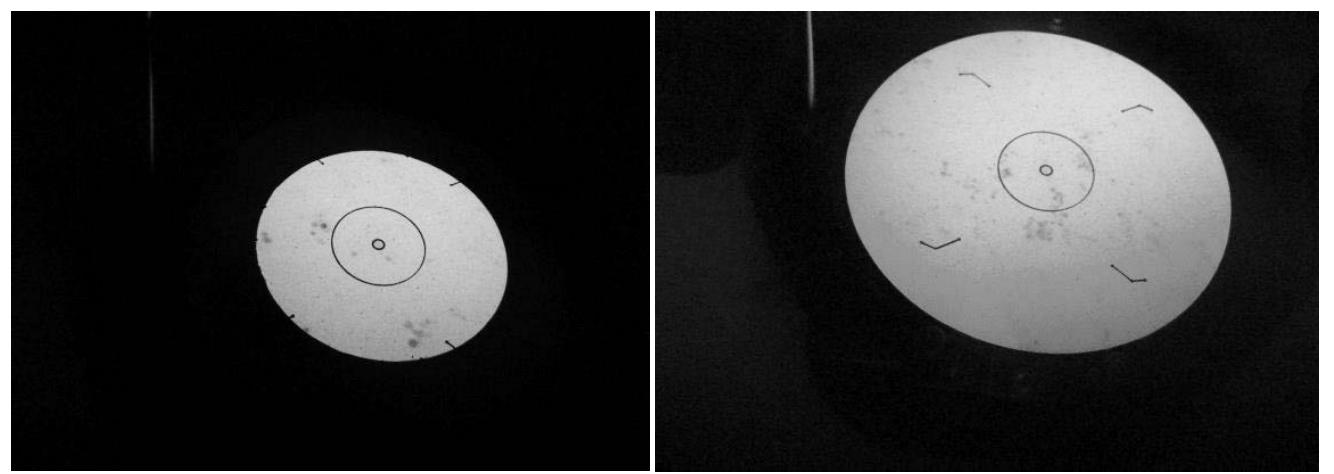

Fig. 4: TEM of optimal montelukast NE formula (F3) 


\section{CONCLUSION}

The present study indicated the possibility to prepare stable nanoemulsion formula containing montelukast sodium with small droplet size and high \% drug release that can be used orally giving a newly easily swallowed dosage form that may improve drug absorption through improving drug solubility and dissolution and may reduce its hepatic metabolism leading to improving its bioavailability that may contribute to reducing its given dose and improves patient's compliance.

\section{ACKNOWLEDGMENT}

The authors would like to thank the university of Al-Mustansiriyah/ Baghdad/Iraq (www. uomustansiriyh. edu. iq) to support the present study.

\section{AUTHORS CONTRIBUTIONS}

All the author have contributed equally

\section{CONFLICT OF INTERESTS}

Declared none

\section{REFERENCES}

1. Harikrishna D, Svitlana S, Feng Z, Jessica M, Galina G, Albert O, et al. Evaluation of a nanoemulsion formulation strategy for oral bioavailability enhancement of danazol in rats and dogs. Pharm Nanotechnol 2013;102:3808-15.

2. Nidhal KM, Anas TA, Zainab HM. Application of the new oroslippery technology in the preparation of enteric slippery coated tablet of naproxen. Int J Pharm Pharm Sci 2017;9:198-204.

3. Zainab HM, Nidhal KM. New easily swallowed tablets with a slippery coating for the antihypertensive drug valsartan. UK J Pharm Biosci 2015;3:9-19.

4. Vivek PC, Dhaval S. A review on novel emulsification technique: a nanoemulsion. J Pharm Toxicol Stud 2017;5:29-37.

5. Halah HA, Ahmed AH. Oral nanoemulsions of candesartan cilexetil: Formulation, characterization and in vitro drug release studies. AAPS J 2017;3:2-16.

6. Setya S, Talegonkar S, Razdan BK. Nanoemulsions: formulation methods and stability aspects. World J Pharm Pharm Sci 2014;3:2214-28.

7. Kriwet K, Muller-Goymann CC. Diclofenac release from phospho-lipid drug systems and permeation through excised human stratum corneum. Int J Pharm 1995;125:231-42.

8. Nirmala MJ, Allanki S, Mukherjee A, Chandrasekaran $\mathrm{N}$. Azithromycin: essential oil based nanoemulsion drug delivery system. Int J Pharm Pharm Sci 2010;5:236-8.

9. Neeharika MS, Jodi BJ. Chronotherapeutics: an optimizing approach to synchronize drug delivery with circadian rhythm. J Cancer Res 2015;2:31-40.

10. Chin Yin C, Hsinchu H. Liquid pharmaceutical foreign application priority data composition of leukotrene antagonsts. United States Patent Application Publication 0147482 A1; 2006.

11. Delmas T, Piraux H, Couffin AC, Texier I, Vinet F, Poulin P, et al. How to prepare and stabilize very small nanoemulsions. Langmuir 2011;27:1683-92.

12. Sarwar BC, Sidharth SJ, Ch NP, Mohammad R, Suryakanta S, Sruti J, et al. Development of solid self-nano emulsifying granules (SSNEGs) of ondansetron hydrochloride with enhanced bioavailability potential. Colloids Surf B 2013; 101:414-23.

13. Beg S. Ultrasonic emulsification of food-grade nanoemulsion formulation and evaluation of its bactericidal activity. Colloids Surf B 2013;101:414-23.

14. Schmolka IR. Artificial skin. Int J Biomed Mater Res 1972;6:571-82.

15. Rajeev S, Gali VS, Pankaj S. Formulation, development and in vitro evaluation of mucoadhesive bilayered buccal patches of montelukast sodium. Int J Pharm Pharm Sci 2012;4:484-97.

16. Thonge SV, Mrudula HB, Harpreet KK, Mandeep D. Taste masked formulation of montelukast sodium for the pediatric population and its evaluation. Asian J Pharm Health Sci 2016;6:1536-49.

17. Mohammad A, Sahabjada, Juber A, Arshad H, Badaruddeen, Arshad M, et al. Development of a new rutin nanoemulsion and its application on the prostate carcinoma pc3 cell line. Excli J 2017;16:810-23.

18. Nidhal KM, Yasser QA. Application of nanoemulsion technology for preparation and evaluation of intranasal mucoadhesive nano-in-situ gel for ondansetron HCl. JGPT 2018;10:431-42.

19. Setya S, Negi P, Razdan BK, Talegaonkar S. Design, development and in vitro investigation of water in oil nanoemulsion for transdermal delivery. World J Pharm Pharm Sci 2014;3:1495-512.

20. Antonio M, Alvarez R, Rodríguez ML. Lipids in pharmaceutical and cosmetic preparations. Grasasy Aceites 2000;51:74-96.

21. Kalra R. Development and characterization of nanoemulsion formulations for transdermal delivery of aceclofenac: research. IJDFR 2010;1:359-86.

22. Gallik S, Rob L, Dean RL. Understanding beer's law: An interactive laboratory presentation and related exercises. JLCE 2014;2:44-9.

23. Hussein AA. Preparation and evaluation of liquid and solid selfmicro emulsifying drug delivery system of mebendazole. Iraqi J Pharm Sci 2014;23:89-100.

24. Sonal S, Poornima N, Razdan BK, Sushama T. Design, development and in vitro investigation of water in oil nanoemulsion for transdermal delivery. World J Pharm Pharm Sci 2014;3:1495-512.

25. Samira K, Fariborz K, Amir A. Design and evaluation of the oral nanoemulsion drug delivery system of mebudipine. Drug Delivery 2016;23:2035-43.

26. Ming Jun T, Yaw Syan F, Yu Hsuan L, Yaw Bin H, Pao Chu W. The effect of nanoemulsion as a carrier of the hydrophilic compound for transdermal delivery. Plos One 2014;9:1-7.

27. Tamilvanana S, Senthilkumarb SR, baskarb R, Thenrajan Raja, Sekharanb TR. Manufacturing techniques and excipients used during the formulation of oil-in-water type nanosized emulsions for medical applications. J Excipients Food Chem 2010;1:11-29.

28. Manuel GM, Philip ES. Flavor and compositional comparison of orange essences and essence oils produced in the United States and in brazil. JAFC 1990;38:799-801.

29. Allen LV, Popovich NG, Ansel HC. editors. Disperse system. Philadilphia: Lippincott Williams and Wilkins Publishing; 2011.

30. Ma TY, Hollander D, Krugliak P, Katz PK. PEG 400, a hydrophilic molecular probe for measuring intestinal permeability. Gastroenterology 1990;98:39-46.

31. Ankur GH, Burak ET, Alan H, Patrick SD. Nanoemulsions: formation, properties and applications. Royal Society of Chemistry 2016:12;2826-41.

32. Beg S. Development of solid self nano emulsifying granules (SSNEGs) of ondansetron hydrochloride with enhanced bioavailability potential. Colloids Surf B 2013;101:414-23.

33. Tadros TF. editor. Emulsion formation, stability, and rheology. Germany: Wiley-VCH Verlag GmbHand Co. KGaA, Weinheim; 2013.

34. Chhabra G, Chuttani K, Mishra AK, Pathak K. Design and development of nanoemulsion drug delivery system of amlodipine besilate for improvement of oral bioavailability. Drug Dev Ind Pharm 2011;37:907-16.

35. McClements DJ. Colloidal basis of emulsion colour. Curr Opin Colloid Interface Sci 2015;7:451-5.

36. Leong TSH, Wooster TJ, Kentish SE, Ashokkumar M. Minimising oil droplet size using ultrasonic emulsification. Ultrason Sonochem 2009;16:721-7.

37. Avachat AM, Patel VG. Self-nanoemulsifying drug delivery system of stabilized ellagic acid-phospholipid complex with improved dissolution and permeability. SPS 2015;23:276-89.

38. Martin AN, Sinko PJ. Martin's physical pharmacy and pharmaceutical sciences. In: Lippincott Williams, Wilkins: Sinko PJ. editors. Interfacial phenomena. 6th edi. London: Lea and Febiger; 2011. p. 365-7.

39. Mathew DS, Juang RS. Role of alcohols in the formation of inverse microemulsions and back extraction of proteins/ enzymes in a reverse micellar system. Sep Purif Technol 2007;53:199-215. 